\title{
Design of Joint Cooperative Routing, MAC and Physical Layer with QoS-aware Traffic-based Scheduling for Wireless Sensor Networks
}

\author{
Jawad Ahmad Haqbeen ${ }^{1}$, Takayuki Ito ${ }^{1}$, Mohammad Arifuzzaman ${ }^{2}$, Takanobu Otsuka ${ }^{1}$ \\ ${ }^{1}$ Department of Computer Science \& Engineering, Nagoya Institute of Technology, \\ Nagoya, Aichi 466-8555, Japan \\ E-mail: jawad.haqbeen@itolab.nitech.ac.jp; ito.takayuki@nitech.ac.jp; Otsuka.takanobu@nitech.ac.jp \\ ${ }^{2}$ Department of Electronics \& Communications Engineering, East West University, \\ Aftabnagar, Dhaka 1212, Bangladesh \\ E-mail:mazaman@ewbd.edu
}

\begin{abstract}
Compare the WSN with other conventional network system, the changing or charging the existing battery of sensor node within WSN is a tough job. Therefore, since last decade the effort has been made to design and introduce a large number of communication protocol for WSN with given concern on the performance parameter of energy efficiency and still the key requirements within WSN domain, that how to incrementally expands the energy minimization consuming techniques of sensor battery. The other parameters include latency, fairness, throughput and delivery ratio. In this work, we propose a novel joint cooperative routing, medium access control (MAC) and physical layer protocol with traffic differentiation based QoS- aware for wireless sensor network (WSNs). This is referred to as a Joint Routing, MAC and Physical layer protocol (J-RMP). By blending the classical layered approach and combining routing, MAC and physical layer functions, the proposed J-RMP protocol achieves a solution for energy efficiency in WSNs. Convention ally, the problem of energy efficient protocol design is considered independently at respective layer of protocol stack. This paper has taken a holistic approach of finding solution by addressing the possible energy gain in Routing, MAC and Physical layer together. Firstly, it is seen that communication path with the large number of short hop substantially minimizes energy consumption. This phenomenon is used while instantaneous network information is collected with minimum overhead through the control packets for selecting next hop. Based on the updated network knowledge the next hop neighbor is chosen with reduced control overhead. Further, we describe how J-RMP protocol uses the approach for finding the constrained shortest path for forwarding packets [1], which results in load balancing in WSNs and provide mathematical analysis of node forwarding path determination within network. Finally, we compare our proposed method with existing protocols by ns-2 simulator and confirm that J-RMP outperform in some cases in term of sensitive traffics. The experimental results have proved that the traffic load has been balanced to a certain traffic, and energy efficiency has been achieved by properly adjusting the applied theory. The outcome supports the theory, and proves the effectiveness of our proposed scheme. This kind of protocol should have significant implications for various WSNs application where energy constraint is the main point of concerns.
\end{abstract}

Keywords: Cross Layer Design, Wireless Sensor Networks (WSNs), Medium Access Control (MAC), Quality of Service (QoS), Energy Efficiency.

\section{Introduction}

Wireless sensor network (WSNs) have become so popular due to its wide range of applications and a significant number of studies are going on in this field.
Generally, sensor nodes and the battery equipped within it are small. And unlike other wireless networks, due to its deployment circumstances it is difficult to re-charge or replace nods battery. Since the prime issue of WSNs which is considered to design a communication protocol 
is energy efficiency. most promising and potential field of application of WSN includes industrial, medical facilities, home appliance and buildings monitoring and control networks [1] [2] [4]. In most of the cases the applications developed by the WSNs is consist of a large number of wireless sensor nodes in a random manner. The life time of sensor notes is important, so energy efficiency plays a vital rule on designing of the protocol of sensor networks [3]. Since prolonging the life time of node is important topic of research for researcher and designers, a large number of research has been going on designing communication protocol for WSN with particular interest given to concern on the performance parameter of energy efficiency. It is because in WS network, sensor nodes do not wake-up time to time rather prefer going to sleep in order to preserve energy as depicted in Fig. 1. Nowadays it has been seen that it is common practise to make a concern on energy efficiency issue by targeting a particular layer of the WSN network architecture. For example, in [5], [16], the authors design routing protocol while targeting energy efficiency parameter. From the literature state of the art of WSNs protocol design it seems that the under layered architecture protocol design is on the way to state of maturity due to lots of concentration on it. Meanwhile, the cross-layer protocol for WSN which is one of desirable approach to achieve and upgrade the performance parameter metrics of protocol design is still under work for its state maturity life cycle. Nevertheless, a number of researcher concentrate on designing cross-layer protocol in the literature [1], [2], [4], [7], [16], which are discussed in section 2 of this paper.

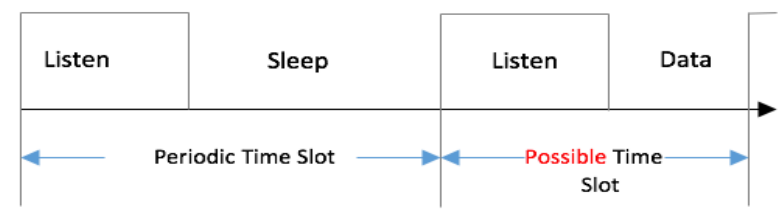

Fig. 1: Periodic timing scheme of sensor node.

In this work, we propose a cross layer protocol together with joint routing, MAC, and Physical (J-RMP) layer for energy efficient wireless sensor network. In J-RMP, we exploit the concept of Received Signal Strength Indicator (RSSI) in physical layer. We calculated the link error rate which is required for transmission the data/packet to the neighbourhood nodes. On the other hand, network layer chooses the possible route for transmitting data packets from source to destination taking the information from the Physical layer. In MAC layer, when a transmission occurs with the acknowledgment (using piggy-back) instantaneous energy information from a node to destination is collected. Finally, from several possible paths the sleep schedule of MAC layer identifies the optimum path to forward the data /packet from source to destination with efficient energy. Thus the protocol J-RMP is a blend of functionalities of Network, MAC and Physical layer in order to achieve higher energy efficiency. Our protocol uses and accommodate the basic novelty features of intelligent hybrid MAC protocol [3] such as link and broadcast scheduling's. The term (rendezvous) used in our paper for link scheduling proposes, it has been defined as a time slot which explicitly dedicated to a pair of senor nodes that has been used for their communication. During link scheduling, rendezvous slot forms a channel for sending (transmission) and receiving (reception) with one of their neighborhood nodes.

In our work, the term channel refers to node time slot. The J-RMP protocol combine the functionalities of intelligent hybrid MAC protocol (IH-MAC) [3] and Routing Protocol for Lossy power and network (RPL) [18]. By integrating the functionalities of IH-MAC and RPL and leveraging the novel features of piggy-backing and modifying the constraint shortest path of network, the J-RMP protocol maximizes the network lifetime in terms of energy efficiency and minimizes delay. The rest of the article is structured as following: We begin by literature reviewing of related work in section 2 . In Section 3, we define the system scenario and network model that we consider for our protocol design. Section 4, we briefly describe the protocol stack of our proposed protocol, talk over path forwarding determination and path selection procedure of J-RMP. In addition, we describe our proposed algorithm for manipulating (updating) sensor node table in this section. In Section 5 , to show the effectiveness of our protocol performance, we conduct power simulation experiments through analyzing the performance of J-RMP with other protocols. Finally, we provide the conclusion and highlight the future research issues in Section 6 of the paper.

\section{Literature Review}

In literature we found that several protocols have been considered the energy efficiency parameter as a main attribute design of a protocol [1], [2], [4], [7], [16]. For example, we focus on the protocol presented in [1], [7] and [16] by authors, as we used to target in our work. In [7], [16] the authors proposed the energy efficient MAC layer protocol for WSNs system. Similarly, in [8-10] authors pay attention to achieve energy efficient network protocol by proposed the design of routing protocol. Some group come up with a proposal of cross 
layer designing concept with a limited range of application. Literature supports the concept of existence of cross layer protocol but this concept is not much more mature for different constrains and it is on the developing phase. According to the authors of [11], they proposed a cross-layer design with optimize framework using the concept of optimization agent that ensure the exchange and control of information between various protocol layers. In [12], the author shows a cross layer design of MAC and Physical layer protocol techniques with better performances in WSNs considering link reliability, energy efficiency, latency, duty cycling, packet recovery, and so on. They also address few drawbacks of cross layer design with MAC and Physical layer protocol like processing of distributions, tradeoff between energy consumption and energy performance, scheduling techniques etc. In [13], the authors proposed a high energy efficient cross layer protocol design, which prefers the more potential packet and avoided the less potential packet for transmission. By thus it increases the network performance by increasing the ratio of more useful packet transmission in network load. So at loading condition the energy efficiency will be improved. Considered as a "Breath", a cross-layer protocol was designed by the authors of [14], which maintained both packet delivery and delay probabilities with minimum energy consumption of the network for the use of control applications in WSNs. In [15], the authors give a way to maximum use of the network. They considered in their design, itself a node find out their routing and transmission power in a dynamic environment. In [16], the authors proposed an Intelligent Cross-Layer protocol for WSNs considered as I-XLP through blending different layer functions and combining functionalities of MAC, routing and congestion control achieved its goal. In [17], the authors proposed a Cross-Layer protocol for WSNs considered the joint optimal design of the physical, Medium Access Control (MAC) and routing layer with Energy Optimization Approach (EOA). The EOA controls the level of transmission power and record it in physical layer in a dynamic fashion. Then network layer utilizes that information of power level of each node and within this time in MAC layer, EOA determine the duty-cycle of each node and control the collision and overhearing problem of the network. And finally designing MAC protocols for WSNs with provision of quality of servicer (QoS) has also been considered as active area of research in WSNs field [21], so we considered it in our protocol as well.

\section{System Network Modeling of J-RMP}

We set a target to apply our proposed design protocol of J-RMP in home appliances for monitoring purpose such as monitoring power consumption of the appliance in the home, reporting alarm in case of crossing the threshold value of smoke, checking water level and take necessary steps to turn on/off the motor etc. In Fig. 2, it shows the high level picture of the scenario.

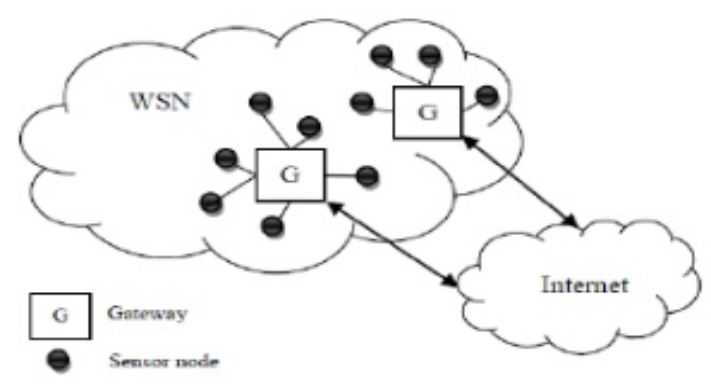

Fig. 2: An Internet-integrated High level view of building monitoring application of WSN

Each sensor node is considered as a relay for its neighbour node. In this situation every quantized data/packet are send from sources to the sink or a group of sinks thorough sensor nodes like multi-hop approach and at the same time each node plays the role as data packet forwarding sensor node for its neighbour sensor node.

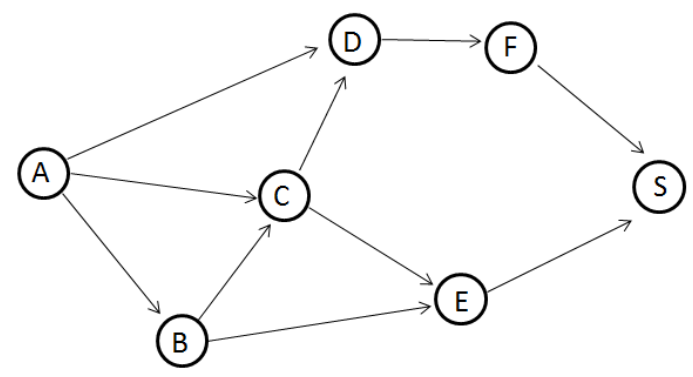

Fig. 3: Assumed Network model, directed Acyclic graph.

Using a Directed Acyclic Graph (DAG), we modelled our system network which is shown in Fig. 3. Here we take,

$$
\begin{aligned}
& \mathrm{G}=(\mathrm{S}, \mathrm{L}) \text { where, } \\
& \quad \mathrm{S}=\text { set of sensor nodes } \\
& \mathrm{L}=\text { set of directed links }
\end{aligned}
$$

Towards the destination node each link from transmitting node to receiving node is representing by $l$. A link $l$ is exists from $i t h$ node to $j t h$ node, if and only if the received power at $j$ th node is higher than a set threshold level, when ith node transmits maximum power with no interference. The topology of network 
modelling with respect of node and link interaction can be represented as $A \in Z^{L * N}$ Link-Node incidence metric. The matrix $n_{i_{\mathrm{th}}}, \mathrm{j}_{\mathrm{th}}$ entry, associated with the link $l_{\mathrm{th}}, \mathrm{j}_{\mathrm{th}}$ is as follows:

$$
\begin{aligned}
& \mathrm{ni}_{\mathrm{th},}, \mathrm{j}_{\mathrm{th}}=\left\{1 \rightarrow \text { the link } l_{\mathrm{t} \text { th }} \mathrm{j}_{\mathrm{th}} \text { exist from } i \text { th node to } j t h\right. \\
& \text { node }\}
\end{aligned}
$$

And If,

$$
\begin{aligned}
& \mathrm{ni}_{\mathrm{th}, \mathrm{j}_{\mathrm{th}}}=\{0 \rightarrow \text { No link exist from ith node to } j t h \\
& \text { node }\}
\end{aligned}
$$

\section{Protocol Stack of J-RMP}

In this section, we briefly explained the design principle of our proposed novel joint routing, medium access control (MAC) and physical (J-RMP) layer functions to achieved our desired goal. To design J-RMP we used several key concepts from IH-MAC [3], RPL [18], RSSI [18] and combining their functionalities. To support both broadcast and link scheduling, the idea of energy efficient MAC protocol (IH-MAC) is used in Physical layer link error rate is calculated using RSSI which is required for transmission the data/packet to the neighbourhood nodes. On the other side, to support IPv6 for resource-constrained devices according to the Internet Engineering Task Force routing protocol for low-power and lossy network (IETF ROLL) working group, RPL has been recently upgraded [18]. DAG routing structure is formed using RPL which is a simple distance vector routing protocol over a physical network calculating a variety of routing combinations.

\subsection{Protocol Stack of J-RMP}

Our proposed frame structure of J-RMP is followed by request-to-send (RTS) or clear-to-send (CTS), and the acknowledgement (ACK) with a fixed length synchronous (SYNC) data period, as depicted in Fig. 4. According to [3-4], synchronization of frame is maintained by virtual clustering. During virtual clustering, sensor nodes can handle different schedule from its neighbour hop and each node is given a unique identification number (ID). The principle of J-RMP is based on slotting the full length communication time and loss of energy into fixed length frame.

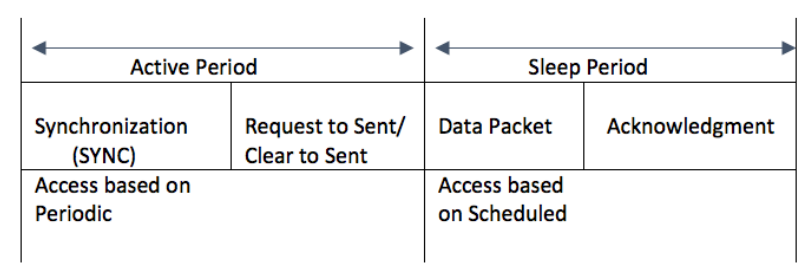

Fig. 4: Slot structure of proposed J-RMP protocol.

Our proposed protocol sort data packet based on their delay features and then store it to reserved queue list. The source sensor node sense the level of data sensitivity based on their importance and accordingly, the application layer applies an extra bit at the end of the packet to set their priority, as depicted in Fig. 5. When will be transmits the data a sensor node first checks critical data and give them priority in transmission. If the sensed send data is critical, the node requests a random back-off within a fixed time period $\mathrm{T}_{\mathrm{f}}$. After expires of $\mathrm{Tf}$, nodes run clear channel assessment (CCA) to check the channel condition and in the situation of clear channel the node transmit data but if the channel is busy then it waits until it's become free and repeats the above process.

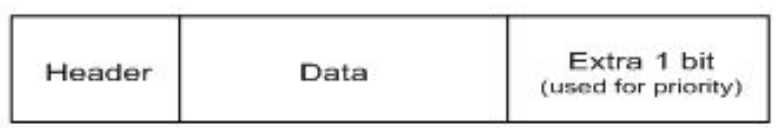

Fig. 5: Structure of each data packet for proposed protocol.

In the case of non critical data, sensor node waits for a time period of $\mathrm{T}_{\mathrm{F}}$ within a maximum contention window $\left[\mathrm{T}_{\mathrm{F}}, \mathrm{T}_{\mathrm{W}}\right]$ and performs random back-off performances. After expires of $\mathrm{T}_{\mathrm{F}}$, nodes run $\mathrm{CCA}$ to check the channel condition and in the situation of clear channel the node transmit data but if the channel is busy then it waits until it's become free and repeats the above process. It should be mention that when a time slot is declared as rendezvous slot the sensor node behaves like a time division multiple access (TDMA) link scheduling. During a sleep state the node switch off its radio and switch On setting the timer. The duration of timer is predefined with consideration of duty cycle and existence rendezvous communication between any pair of neighbour nodes as depicted in the Fig. 6. When the timer time span ends, the node come to wake-up state. It switches on its radio and goes to listen to the data channel and leads to idle listening state then. In case the sensor node receives any sending or receiving signal it goes in the CSMA/CA state, else after time out it goes to sleep state. If the sender sensor node wins the contention both the intended sender and receiver go to the $\mathrm{Tx} / \mathrm{Rx}$ state and go to sleep state after successful communication. Sensor nodes that failed to fulfil contention go to sleep state. 


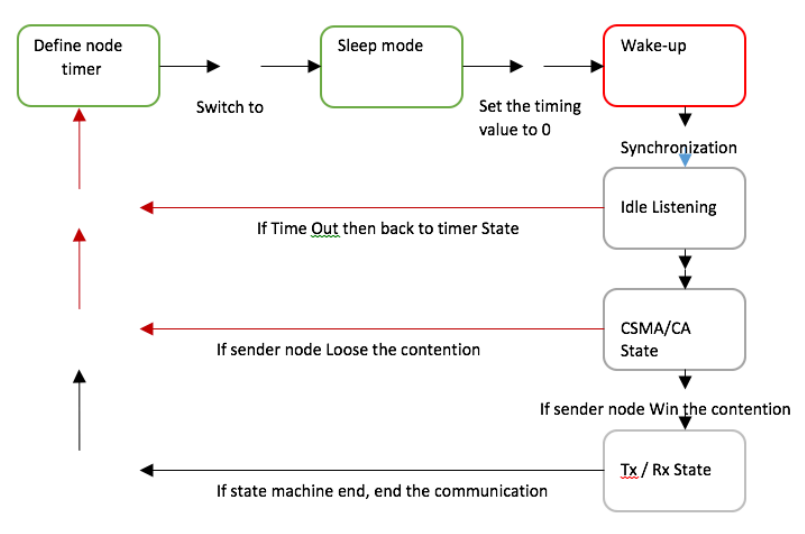

Fig. 6: State Machine six modes of sensor nodes working in JRMP.

\subsection{Forwarding Path Determination}

In this section, we discuss about the data forwarding path determination process in J-RMP by the aid of shortest constrained path, shortest energy path and shortest delay path [1]. Both link and node constrained is considered to choose the shortest path in J-RMP. In the J-RMP associated with constraint based routing, which is one of best path determination technique in our protocol. The constraint applies to both link and sensor nodes. In case the link or sensor node does not match the requested shortest constraint path, then it is pruned from nominated neighbour set and go towards constraint shortest path.

\subsection{The technique of sensor Node Constrained in J- $R M P$}

For node constraint in J-RMP, we considered the packet forwarding rate threshold [18] to find the availability of receiving additional forwarding packet. At each node, the overall input packet rate can be found by adding generated packet rate at that particular node and summation of forwarding packet rate from all neighbour nodes towards the node. Now the overall output packet rate of each node can be calculated as the sum of the generated packet rate of that particular node, the overall forwarding packet rate of the node and the packet error rate. The overall input packet at node $i, \lambda_{i}$ can be represented as:

$$
\lambda_{i}=\lambda_{i, i}+\lambda_{i, \text { relay }}=\lambda_{i i}+\sum_{j \in N_{i}^{i n}} \lambda_{j t h, i t h},
$$

Where $i$ is the sequence common number, $\lambda_{i i}$ the higher generated data rate, $\lambda_{j t h, i t h}$ is the forwarding data rate from $i_{\text {th }}$ node to $j_{t h}$ node, $N_{i}^{i n}$ is the set of sensor node which $i_{t h}$ node receives forwarding packet. The $i_{\text {th }}$ node aims to send all the data in its buffer. Thus the overall data output rate of $i_{\text {th }}$ node can be given as follows:

$$
\mu_{i}=\left(\lambda_{i, i}+\lambda_{i, f w d}\right)+e_{i}\left(\lambda_{i, i}+\lambda_{i, f w d}\right) .
$$

Where $\lambda_{i, f w d}$ is the overall forwarding data rate of ith node and $e_{i} \mathrm{~h}$ is the data error rate. Thus, $e_{i}\left(\lambda_{i, i}+\right.$ $\left.\lambda_{i, f w d}\right)$ can be used to calculate the data retransmission. The $\lambda_{i, f w d}$ is given as follows:

$$
\lambda_{i, f w d}=\sum_{\substack{1 \leq \mathrm{jth} \leq N \\ \mathrm{ith} \neq \mathrm{jth}}} \lambda_{j t h, i t h} \mathrm{n}_{\mathrm{Av}}(\mathrm{ith}, \mathrm{jth})
$$

Now let's consider $\mathrm{T}_{\mathrm{RX}}$ and $\mathrm{T}_{\mathrm{TX}}$ as average receiving and transmitting time of $i_{t h}$ node, and $\mathrm{T}_{\mathrm{TL}}$. Hence, from equation (1) and (2), we get following two more equations:

$$
\begin{aligned}
T_{T X}=[(1 & \left.+e_{i}\right)\left(\lambda_{i, i}\right. \\
& \left.\left.+\lambda_{i, f w d}\right)\right] T_{T l} T_{T X(\text { avg })},
\end{aligned}
$$

and

$$
T_{R X}=\left[\lambda_{i, f w d}\right] T_{T l} T_{T X(a v g),},
$$

The average time of a node spends in transmitting and receiving can be found with a minimum interval from the overall input and output of packets rate. Every node has a duty cycle with a limited time span and during this time the generated and received packets should be transmitted, otherwise there will be a backlog on that node.

Now in order to stop backlogging of sensor node we should transmit the generated and received data during the given active period of sensor node duty cycle. Let's consider a micro time (second) span of $T_{T l}$. Hence, the given active period of sensor node duty cycle considered as $\delta T_{T l}$, where $\delta$ indicates the duty cycle. Thus the $\delta T_{T l}$ given as follows:

$$
\begin{gathered}
\delta T_{T l} \geq\left[\left(1+e_{i}\right) \lambda_{i, i}+\left(2+e_{i}\right) \lambda_{i, f w d}\right] \times \\
T_{T l} T_{T X(\text { avg })},
\end{gathered}
$$

The $T_{T X(a v g)}$ in (6) is the average transmitting time required for frame/packet after receiving with one of their neighbourhood nodes, and the queuing time and MAC overhead exist in it. So in order to determine the availability of node in a path we consider following:

$$
\lambda_{i, f w d} \leq \lambda a d_{i, f w d}
$$

where

$$
\begin{aligned}
\lambda a d_{i, f w d}=1+ & \frac{\delta}{\left(2+e_{i}\right) T_{T X(a v g)}} \\
& -\frac{\left(1+e_{i}\right)}{\left(2+e_{i}\right)} \lambda_{i, i} .
\end{aligned}
$$




\subsection{J-RMP Node Energy Constraint}

Energy constraint in J-RMP is directly connected with system network lifetime, the energy of node within WSN network is defined as a energy the WSN system depend to be active with it, the time span the energy of last node die within a network, the time the life of a system end to operate anymore. Let the $R m_{e(i)}(t)$ be the available remaining energy node $i$ at the time $(t)$ and $C n_{e(a v g) i, j}$ represent the transmitting average energy consumption of node $i$ th to node $j t h, \mathrm{f}$ is number of data bits per slot/frame, $\in$ is the extra energy consumption overhead for node $i$ th before initiating the transmission such as idle listening and transition state and $\gamma$ is the index of energy balance used to rotate the forwarding path among other sensor nodes. Then the energy availability of node $i$ in the node path will be determined as follows:

$$
R m_{e(i)}(t) \geq\left(\sum_{\substack{1 \leq \mathrm{jth} \leq N \\ \mathrm{ith} \neq \mathrm{jth}}} \mathrm{e}_{\mathrm{ith}, \mathrm{jth}} \mathrm{n}_{\mathrm{AV}}(\mathrm{ith}, \mathrm{jth}) \mathrm{S}+\epsilon\right) \gamma
$$

\subsection{J-RMP Link Constraint}

Link constraint in J-RMP is defined by performing link schedule with a particular neighbour and in this case the node has not reached the threshold value of the incoming traffic and perform link scheduling with its dedicated neighbour sensor node [3].

The J-RMP protocol considers the signal strength attenuation, let the transmitted signal amplitude is $\mathrm{T}(\mathrm{t})$, the received signal amplitude is (r) at distance $\mathrm{D}$ from the transmitter and $\alpha$ is a parameter value ranging from 2 to 4 and considered the proportionality constant $\mathrm{k}=$ 1 without considered loss generality then J-RMP follows the following equation:

$$
T(r)=K T(t) D^{-\alpha} .
$$

J-RMP protocol selects path that comprises more sensor with small distance between them. That is if a path has less sensor nodes but the longest distance between the nodes is large that path is not preferable.

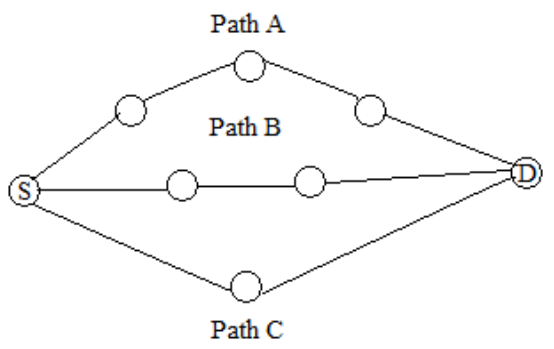

Fig. 7. Different data transmission paths from source to destination.
In fig.7, path $\mathrm{A}$ is more preferable than other two path $B$ and path $C$, provided that other constraints (MAC layer and network layer functions) meet.

\subsection{J-RMP Shortest Power and Delay Path Selection Procedure}

In this subsection, we discuss how to find shortestpower gain path within our network and defined as a path that offering the lest energy consumption. This shortest power is determined by solving (7) and (9) problem.

$$
\begin{aligned}
& \min \sum_{S \in N} R m_{e(i)}(t) \\
& \quad \lambda_{i, f w d} \leq \lambda a d_{i, f w d} \\
& s \in N \backslash N^{\prime} ; \\
& R m_{e(i)}(t) \\
& \geq\left(\sum_{\substack{1 \leq \mathrm{jth} \leq N \\
\mathrm{jth} \neq \mathrm{i}}} \mathrm{e}_{\mathrm{ith}, \mathrm{jth}} \mathrm{n}_{A V}(\mathrm{ith}, \mathrm{jth}) \mathrm{S}+\in\right) \gamma
\end{aligned}
$$

Where $\mathrm{s}$ is set of all intermediate sensor nodes towards the sink node and $\mathrm{N}$ will be the set of sensor nodes participating in the link scheduling.

And shortest delay path in our protocol defined as the path offering the shortest end to end delay in the network, and delay will be calculated at each hop and piggy backed to the source sensor node through Acknowledgment (ACK) field of the MAC frame. Let the $d_{i}$ be the delay generated due to the random backoff take by the sensor nodes before running CCA, and $n_{i}$ represents the delay generated if the node belongs out of their cluster, and $q_{d}$ represent the queuing delay. Then, destination estimated time can be calculated by summing up the latency at each hop $n_{h}$, towards the path to the sink node.

$$
\operatorname{DET}\left(n_{h}\right)=\sum_{i=1}^{n_{h}} d q+d_{i}+n_{i}
$$

Therefore, this shortest delay in our protocol is determined by solving (7) and (9) problem as well.

$$
\begin{gathered}
\min \sum_{A}^{n_{h}} d q+d_{i}+n_{i} \\
\lambda_{i, f w d} \leq \lambda a d_{i, f w d} \\
s \in N \backslash N^{\prime} ; \\
R m_{e(i)}(t) \geq \\
\left(\sum_{1 \leq j \mathrm{jth} \leq N} \mathrm{e}_{\mathrm{ith}, \mathrm{jth}} \mathrm{n}_{A v}(\mathrm{ith,jth}) \mathrm{S}+\in\right) \gamma
\end{gathered}
$$




Algorithm 1 Pseudo code for manipulation of sensor
node table

1: procedure REQUEST $\left(\mathrm{e}_{\mathrm{ET}}, \mathrm{d}_{\mathrm{ET}}, \mathrm{e}_{\mathrm{RM}}, \mathrm{n}_{\mathrm{AV}}\right)$

$\triangleright$ Estimated energy $\mathrm{e}_{\mathrm{ET}}$, Estimated delay $\mathrm{d}_{\mathrm{ET}}$, Remaining energy $\mathrm{e}_{\mathrm{RM}}$ and Node availability $\mathrm{n}_{\mathrm{AV}}$

2: m: Matrix $\left(\mathrm{e}_{\mathrm{ET}}, \mathrm{d}_{\mathrm{ET}}, \mathrm{e}_{\mathrm{RM}}, \mathrm{n}_{\mathrm{AV}}\right)$

3: $\mathrm{N}(\mathrm{ith}, \mathrm{jth})$ : updating node table at sensor node $n_{\mathrm{i}}$ for node $n_{\mathrm{j}}$

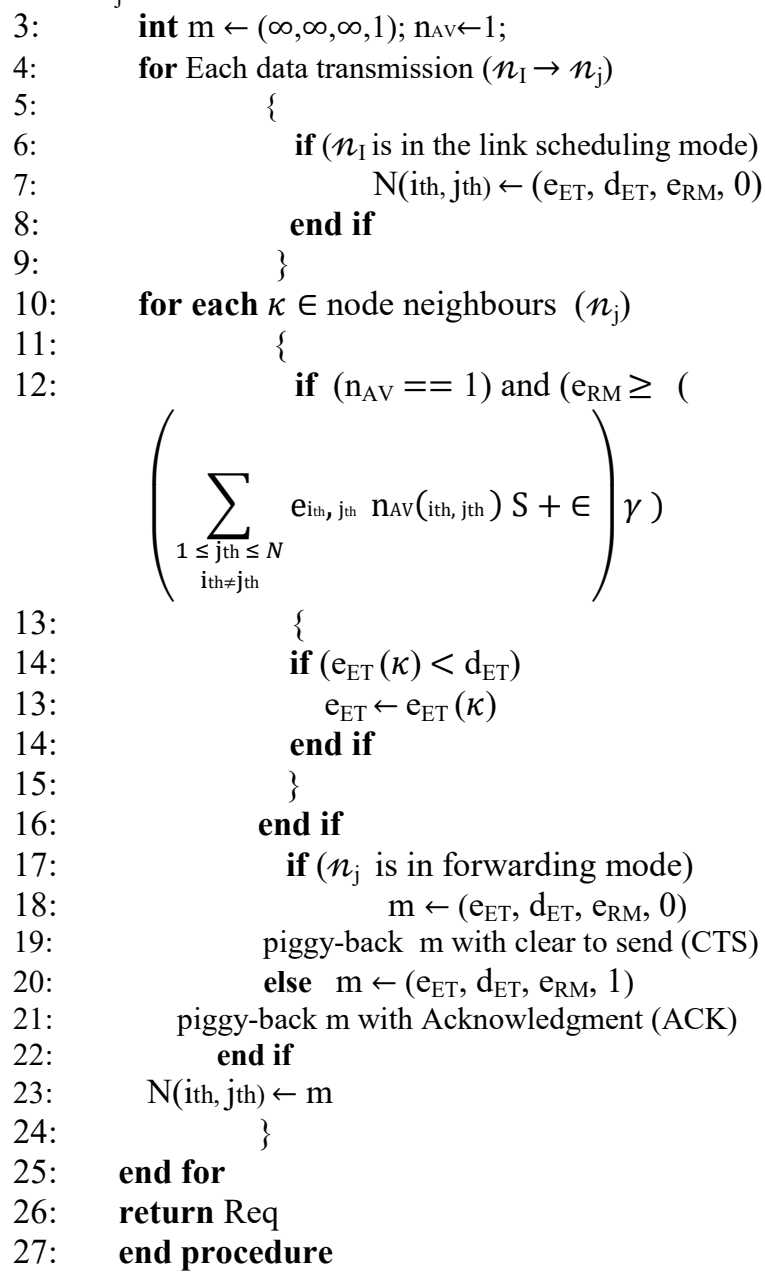

\subsection{J-RMP Path Selection Procedure}

In this subsection, we briefly describe that how the MAC operation assist the routing technique of J-RMP in the appropriate sensor path selection. To explain the JRMP protocol path selection procedure, we assume a simple network model which is seven node Directed Acyclic Graph (DAG), as depicted in fig 2. Every node edge from DAG has weight metrics of power consumption and delay values from sender (source sensor node) to a receiver (destination sensor node). The calculation of multi-hop path values is done through addition. For example, we define each node edge DAG as follows:

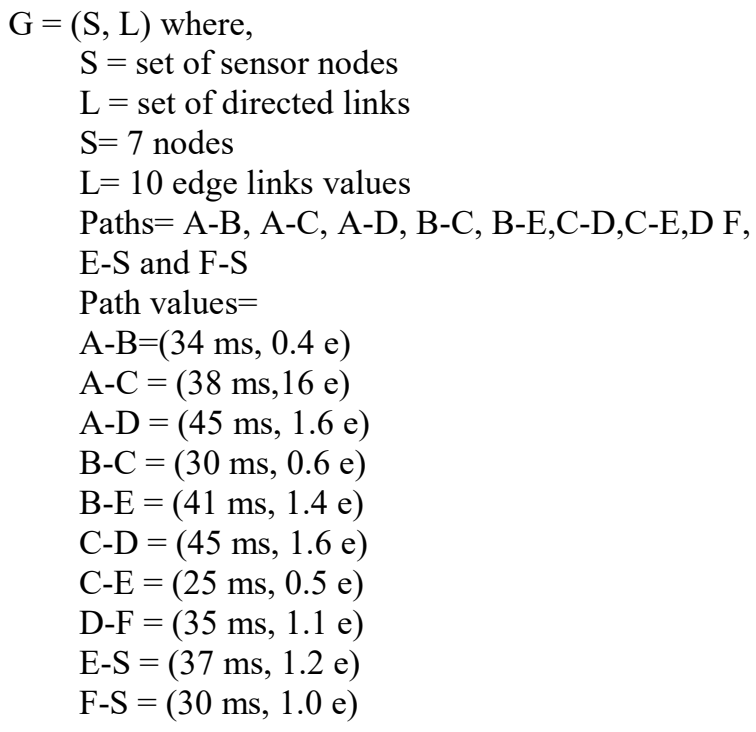

Table 1. Edge weights.

\begin{tabular}{|c|c|c|c|c|c|c|c|}
\hline $\begin{array}{c}\text { Node } \\
\text { edge } \\
\text { weights } \\
(\mathrm{t}, \mathrm{e}) \\
\end{array}$ & A & B & C & D & $\mathrm{E}$ & $F$ & $S$ \\
\hline A & $100,2.7$ & $\begin{array}{c}34,0.4 \\
1 \\
0 \\
0 \\
\end{array}$ & $\begin{array}{c}38,1.6 \\
0 \\
1 \\
0 \\
\end{array}$ & $\begin{array}{c}45,1.6 \\
0 \\
0 \\
1 \\
\end{array}$ & $\begin{array}{l}75,1.8 \\
63,2.1\end{array}$ & $80,2.7$ & $\begin{array}{l}112,3.0 \\
100,3.3 \\
110,3.7\end{array}$ \\
\hline B & 34.04 & $78,2.3$ & $\begin{array}{c}30,0.6 \\
0 \\
1 \\
1\end{array}$ & $\begin{array}{c}75,2.2 \\
1 \\
0\end{array}$ & $\begin{array}{c}41,1.4 \\
1 \\
0 \\
1\end{array}$ & $110,3.3$ & $\begin{array}{l}78,2.6 \\
140,4.3 \\
92,2.3\end{array}$ \\
\hline C & $38,1.6$ & $30,0.6$ & $62,1.7$ & $\begin{array}{l}45,1.6 \\
0 \\
1 \\
\end{array}$ & $\begin{array}{l}25,0.5 \\
1 \\
0 \\
\end{array}$ & $80,2.7$ & $\begin{array}{l}62,1.7 \\
110,3,7 \\
\end{array}$ \\
\hline D & & & & $65,2.1$ & & & $65,2.1$ \\
\hline $\mathrm{E}$ & & & & & $37,1.2$ & & $37,1.2$ \\
\hline $\mathrm{F}$ & & & & & & $30,1.0$ & $30,1.0$ \\
\hline $\mathrm{s}$ & & & & & & & 0,0 \\
\hline
\end{tabular}

Now let's analyse the optimal predicted edge weight (time, energy) for a path listed in Table 1. In above table the digit "1" and "0" interpreted as "ON" and "OFF" mode for path selection respectively. The J-RMP router process this option to help maintain the efficient path to route. In order to find the cost of transferring a packet from node $\mathrm{A}$ to node $\mathrm{B}$ in fig.3, it requires 34 millisecond delay and 0.4 unit of energy. Therefor, a packet transferring from node $\mathrm{B}$ to sink node $\mathrm{S}$ requires $41+37$ delay and $1.4+1.2$ unit of energy. Thus, from sensor node $\mathrm{A}$ to sensor node $\mathrm{S}$ through sensor node $\mathrm{B}$ the required time, energy metrics is $(34+41+37$, $0.4+1.4+1.2)=(112,3.0)$ or we can say for the cost on 
path A through B to sink $\mathrm{S}$ is $(112,3.0)$ or A-B-E-S = $(112,3.0)$. However, if packet follows sensor node A to sensor node $\mathrm{S}$ through node $\mathrm{C}$, then the required time and energy will be different and the time, energy metrics for A-C-E-S is $(38+25+37,1.6+0.5+1.2)=(100$, 3.3). And if packet follows sensor node $A$ to sensor node $\mathrm{S}$ through node $\mathrm{D}$, then the required time and energy will be different and the time, energy metrics for A-D-F-S is $(45+35+30,1.6+1.1+1.0)=(110,3.7)$. So the minimum edge weight time, energy metrics for node $\mathrm{A}$ is $(100 \mathrm{~ms}, 3.0 \mathrm{e})$. If the objective goal is to be energy efficient and gain minimum energy, then the path that constitute less energy consumption A-B-E-S will be chosen as routing path for packet but if the desire goal is packet delay minimization, then a path with minimum delay A-C-E-S will be selected. Thu, our protocol serves for shortest path for data routing.

Now, how our J-RMP serves the shortest path, we briefly describe it here. For example, we take the case of A-B-E-S. If a sensor node A wants to send a packet to sink node through node $\mathrm{B}$, while sensor node $\mathrm{B}$ already reached the forwarding threshold, here sensor node $\mathrm{B}$ send back the information to sensor node A through either with CTS (it says that I am already in forwarding threshold mode) or through ACK (mean that I already reached threshold mode with receiving of one or more additional data packet). Therefore, based on node edge time, energy cumulative weight metrics, sensor node A select the best choice/path between remaining neighbours path (A-C-E-S through C node) or (A-D-F-S though D node). Similarly, sensor node B is pruned or executed from the path list if it performs the link scheduling mode.

It is worth mention that J-RMP protocol can be extended to achieve other desire goals of protocol performance metrics, such as packet deliver ratio and bandwidth. Furthermore, in our future research, multiplication techniques can be used to integrated the link cost for multi-hop path cost calculation features.

\section{Evaluation and Experimental Results}

In this section, we present the simulation results for our proposed protocol, which demonstrate the effectiveness of our scheme. We evaluated the performance of proposed protocol for the energy efficiency, duty cycle and delivery ratio with other existed protocols. In addition, we compare the JRM, I-XLP and RPL+IHMAC with that of proposed scheme. Throughout the simulation experiments, we vary packet generation interval from $1 \mathrm{sec}$ to $10 \mathrm{sec}$ while applying the average of 8 experiments for each parameter value. We describe the experiment and result in detail as following.

\subsection{Simulation Data and Setup}

We evaluated the proposed protocol using network simulator ns-2. In the simulation setup, we took 300 sensor nodes which deployed on an area grid of $100 \mathrm{~m}$ $\times 100 \mathrm{~m}$. The nodes are static and are distributed in a uniformly random way on the grid. Each sensor has transmission range of $20 \mathrm{~m}$. The sink node is chosen on the bottom right corner of the grid. The size of a data packet is 30 bytes and the data transmission rate is set to $250 \mathrm{kbps}$. The generation interval varies from 1 to 10 seconds. The results are shown both for the prioritized traffic and non-prioritized traffic. The parameters of our experiments are defined in Table 2, which are consistent with those from [4].

\subsection{Algorithm Description and Implementation}

In order to update the information of routing table between sensor node corresponding to forwarding path determination technique in J-RMP, we create the pseudo code that works as mentioned in algorithm 1. As J-RMP support constraint based routing, where constraints apply both to node constraint and link constraint. It is worth mentioned that nodes energy constrain which is one type of node constraint is applied in our protocol targeted in our algorithm. The definition of our algorithm is as follows. We take a Destination Oriented Directed Acyclic Graph (DODAG) with a set of seven sensor nodes in it, we modelled our system network. The routes are organized along a Destination Oriented Directed Acyclic Graph (DODAG). Towards the destination node each link from transmitting node to receiving node is representing by $l$. A link $l$ is exists from $i t h$ node to $j$ th node, if and only if the received power at jth node is higher than a set threshold level, when ith node transmits maximum power with no interference. We take a Link-Node incidence matrix which include estimated energy consumption, estimated delay, remaining source of energy and node availability as $\mathrm{e}_{\mathrm{ET}}, \mathrm{d}_{\mathrm{ET}}, \mathrm{e}_{\mathrm{RM}}, \mathrm{n}_{\mathrm{AV}}$ respectively. Let the $R m_{e(i)}(t)$ be the available remaining energy node $i$ at the time $(t)$ and $C n_{e(a v g) i, j}$ represent the transmitting average energy consumption of node $i$ th to node $j t h, \mathrm{~S}$ is number of data bits per slot/frame, $\in$ is the extra energy consumption overhead for node $i$ th before initiating the transmission such as idle listening and transition state and $\gamma$ is the index of energy balance used to rotate the forwarding path among other sensor nodes neighbourhoods. After running the algorithm with above definition, If the link or node satisfy specific predefined constrained then the information of routing table update based on shortest constrained path based of J-RMP within network, else it is pruned from the candidate neighbour of sensor node set, Thus the 
algorithm help to update and lead to shortest constrained path within network.

\subsection{Evaluation Protocols}

We compare the performance of the proposed J-RMP with combined layered protocol IH-MAC+RPL, JRM and I-XLP, which are cross layer protocols. The performance metrics used during our protocol evolutions are energy consumption, latency and delivery ratio.

(a) JRM: JRM is a novel joint routing and medium access control protocol with traffic differentiation based on Quality of Service (QoS) for Wireless Sensor Network. Moreover, we did consider the QoS for the comparison.

JRM [1], is another energy efficient cross layer with traffic differentiation based QoS for WSN that we targeted as evaluating the performance of our protocol.

(b) RPL+ IH-MAC: RPL+ IH-MAC: We use RPL and IH-MAC as a layered approach to compare its performance with that of the proposed J-RMP protocol. RPL [18], is routing protocol for energy friendly and lossy networks and IH-MAC [3], is a low power hybrid MAC protocol, so the idea of combining these two protocol is to get them as layered approach to compare it with proposed cross layer scheme (J-RMP).

(c) I-XLP: The last compared protocol is I-XLP [4], which is an energy efficient cross layer protocol with traffic differentiation based QoS aware protocol. W used our previous work, I-XLP as a comparison because we did consider QoS for I-XLP as well.

We simulate both cases, regular traffic and prioritized traffic for the case of experiments 2 (performance metrics of latency). For regular traffic, there is no any priority given for the traffic rate. In the case of prioritized traffic simulation, we considered a five percentage of the total simulated traffic during our simulation experiments. The performance metrics used during our protocol evolutions are energy consumption, latency and delivery ratio.

\subsection{Experimental Results}

The results of experiment 1 , and 2 are illustrate in Fig. 8(a), Fig. 8(b), Fig. 9(a) and Fig. 9(b), correspondingly. For all low traffic (regular traffic) our proposed protocol outperformed compare other protocols. In the first experiment, we conducted experiment for energy consumption under different non-prioritized traffic (just regular traffic). In the second experiment, we run the simulation for packet latency under the different nonprioritized traffic and run $5 \%$ prioritized traffic. On the other hand, the protocol performs equal for high traffic. In our all experiments follows that with increase of traffic value the duty cycle and energy consuming increase.

1) Experiment 1: The results of average energy consumption efficiency vs duty cycle and packet arrival rate are illustrated in in Fig. 8(a) and 8(b), correspondingly. As we see in the Fig. 8(a), the consumption of energy increases with increase of duty cycle accordingly. But it clearly shows that the performance of our proposed J-RMP outperform other two protocols for the lower values $(0-42.2 \mu \mathrm{w} / \mathrm{bit})$ of duty cycles. However, as the duty cycle increase the performance of J-RMP for consuming energy is more evident or equal to some cases compared to other two protocols [1], [3] + [18], [4]. This is because, with the increase of packet arrival rate in RMP, the nodes can not match the physical layer functionalities with other two layers more frequently. The RMP protocol load distribution feature does not support offering much energy gain during consumption; thus, for high duty cycling values all evaluated protocols performance is almost equal. However, with the increase of duty cycling, the J-RMP Link Quality Assessment (LQA) features improved; and it helps the possibility of data packet forwarding easiness to be enhanced through appropriate path route, which we previously described in section 4.3.

So the J-RMP outperform other three protocols for all values of duty cycles. However, when the duty cycle increased the performance of J-RMP is more evident to equal based compared to other three protocols. This is because, with the increase of duty cycle the nodes can match the physical layer functionalities with other two layers more frequently.

In second case of experiment (energy vs packet arrival rate), we can see that the energy consumptions increase with decreasing values of packet traffic for all evaluated protocols. The performance of all evaluated protocol for high values of packet traffic is almost same, this is because all evaluated protocols use the link scheduling features. But as the packet arrival traffic rate more increasing the performance of J-RMP is not efficient. This is because, with the increase of packet arrival rate the nodes can not match the physical layer functionalities with other two layers more frequently. However, as the packet arrival traffic rate decrease the J-RMP outperform compare to [3] $+[18]$, this is because of RPL control signalling overhead, and it let our proposed J-RMP to be more evident compare other protocols in terms of low values traffic packet rate.

2) Experiment 2: The result in the Fig. 9(a) and 9(b), illustrate the compressions of average latency vs duty cycle and packet arrival rate of proposed J-RMP with layered protocols in the case of both regular and 
prioritized traffic, respectively. In case of latency, JRMP outperform other three protocols. Our proposed protocol serves the regular traffic with less delay compare other evaluated protocols. But in terms of prioritized traffic J-RMP perform equally as others. It is because the slight gain of our protocol comes with lower control packet. In Fig. 5(a), we can see that our protocol serves better, it is because, while the RPL routing layer chooses a sensor node neighbour for data transmission, at the same the MAC layer can not match data transmission between sender sensor node and receiver sensor node. So it let additional delay for layered approach RPL.

Table 2. Simulation parameters

\begin{tabular}{c|c}
\hline Parameters & Value \\
\hline Transmission energy & $24.75 \mathrm{~mW}$ \\
Receive energy & $13.5 \mathrm{~mW}$ \\
Idle energy & $13.5 \mathrm{~mW}$ \\
Sleep state & $15 \mu \mathrm{W}$ \\
Frame length & $1 \mathrm{sec}$ \\
Data transmission & $250 \mathrm{kbps}$ \\
Prioritized Traffic & $5 \%$ \\
Packet generation Interval & $1-10 \mathrm{sec}$ \\
Channel Bandwidth & $30 \mathrm{bytes}$ \\
Data Packet length & $100 \mathrm{bytes}$ \\
Control Packet length & 20 bytes \\
Simulation time & 30 days \\
\hline
\end{tabular}

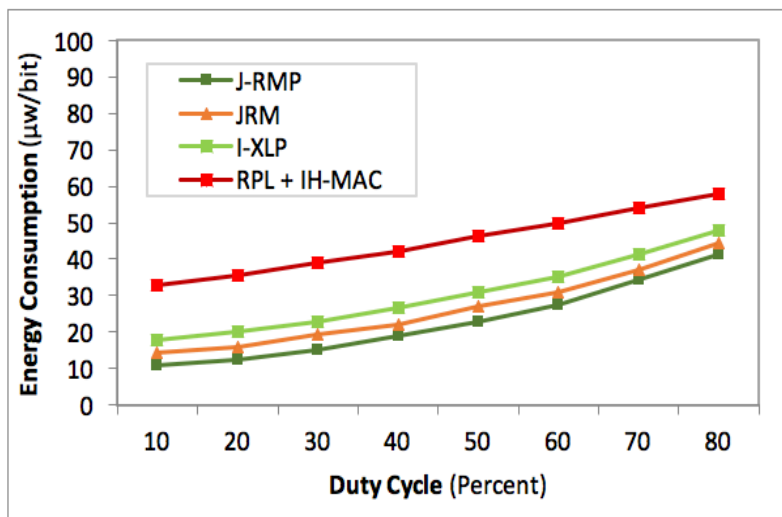

Fig. 8(a): Average energy efficiency vs. duty-cycle.

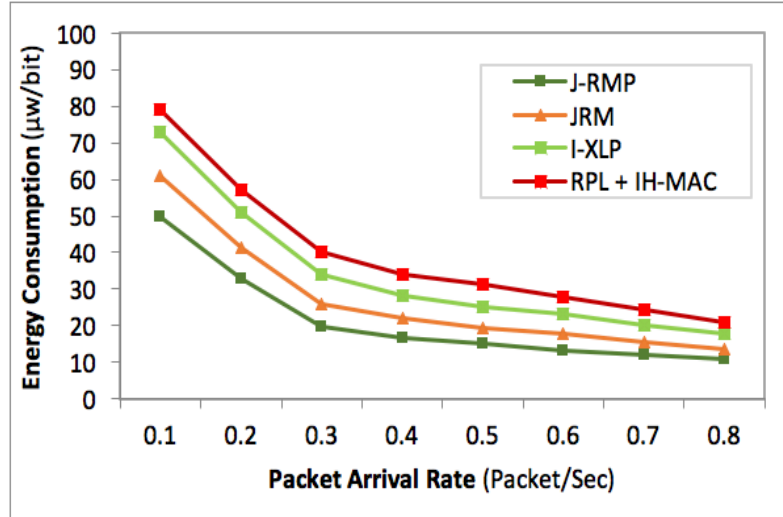

Fig. 8(b): Average energy efficiency vs. packet arrival rate.

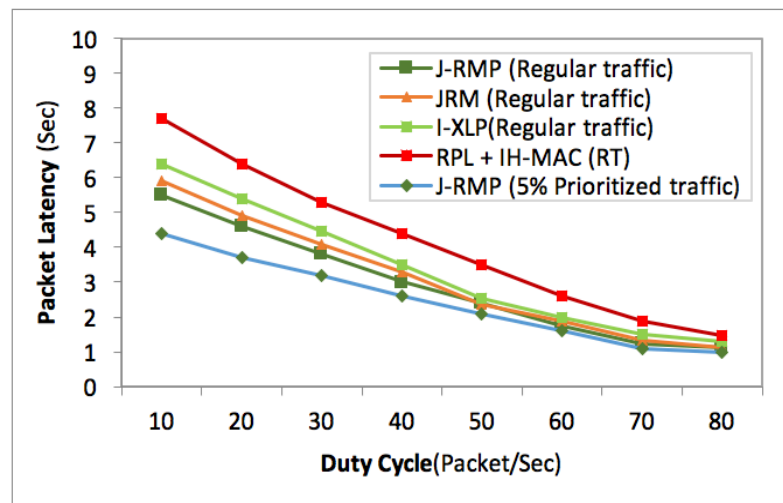

Fig. 9(a): Average latency vs. duty-cycle.

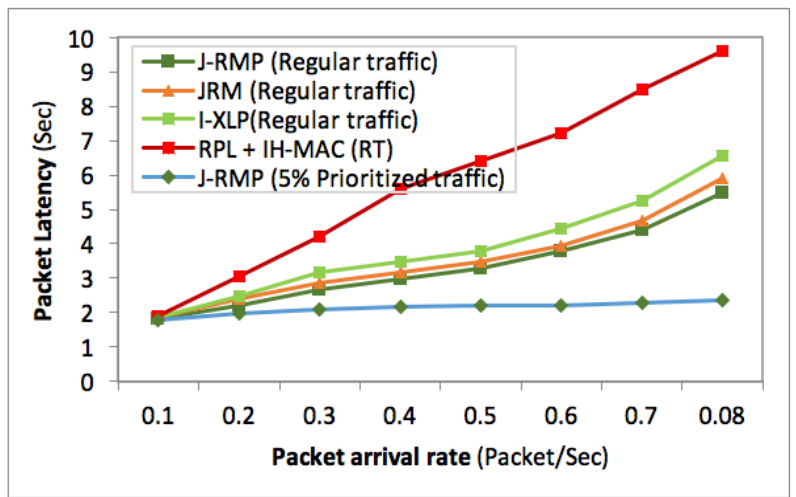

Fig. 9(b): Average latency vs. packet arrival rate. 


\section{Conclusions}

In this paper, we focused on the importance of energy efficient communication protocol with different protocol attributes and proposed a novel energy efficient joint routing, medium access control (MAC) and physical layer protocol (J-RMP) for wireless sensor network (WSNs). Because it is importance to know the characteristics of layer collaborations for efficient energy consumption in the network, demands required for different layers' function optimization. By blending the classical layered approach and combining routing, MAC and physical layer functions, the proposed J-RMP protocol achieves a solution for energy efficiency in WSNs. This paper has taken a holistic approach of finding solution by addressing the possible energy gain in Routing, MAC and Physical layer together. We described our algorithm for the proposed scheme protocol, which preforms the information manipulation task (mainly updating) of sensor nodes within network. It is seen that communication path with the large number of short hop substantially minimizes energy consumption. This phenomenon is used while instantaneous network information is collected with minimum overhead through the control packets for selecting next hop. Based on the updated network knowledge the next hop neighbour is chosen with reduced control overhead. Besides, the J-RMP protocol uses the approach for finding the constrained shortest path for forwarding packets, which results in loading balancing in WSNs. Our protocol performance evaluation and experiments showed that the proposed protocol supresses energy efficiency vs duty cycle and packet arrival as compered with other protocols and incrementally minimize the energy consumption for WSNs. Therefore, the proposed scheme matches the demand of energy efficient communication protocol more optimally. However, J-RMP protocol load distribution feature does not offer much energy consumption gain; thus, our protocol performs same as other evaluated protocols.

\section{Future Works}

As a future work, we plan to work on detail mathematical analytical modelling of proposed scheme and study more sophisticated cases of discrepancy, if it matches and exist with proposed scheme. Another direction of our future work is to study other attributes of protocol such as throughput and delivery ratio. Moreover, there is scope to work on physical layer functionalities optimization with other two layers and improve the efficiency of J-RMP other protocol performance attributes and design energy efficient protocol for other application spaces of WSNs and implement it towards real-world WSNs application.

\section{References}

1. M. Arifuzzaman, O. A. Dobre, M.H. Ahmed, and Telex M.N. Ngatched, "Joint Routing and MAC Layer QoS Aware Protocol for wireless sensor Networks," Global Communications Conference (GLOBECOM), 2016 IEEE, 4-8 Dec. 2016.

2. T. Watteyne, A. Molinaro, M. G. Richichi, and M. Dohler, "From manet to ietf roll standardization: A paradigm shift in wsn routing protocols," IEEE Commun. Surv. Tutorials, vol. 13, no. 4, pp. 688-707, Apr. 2011.

3. M. Arifuzzaman, M. Matsumoto, and T. Sato, "An intelligent hybrid MAC with traffic-differentiation-based QoS for wireless sensor networks," IEEE Sensors J., vol. 13, no. 6, pp. 2391-2399, Jun. 2013.

4. J. A. Haqbeen, T. Ito, M. Arifuzzaman and T. Otsuka, "An Intelligent Cross layer QoS-Aware Protocol with traffic differentiation based for energy efficient communication in WSNs," International Jouranl of Networked and Distributed Computing, vol. 5, no. 2, pp. 80-92, Apr. 2017.

5. G. Wang, K. Wu, and L. M. Ni, "CSMA/SF: Carrier sense multiple access with shortest first," IEEE Trans. Wireless Commun., vol. 13, no.3, pp. 1692-1702, Mar. 2014.

6. M. Arifuzzaman and M. Matsumoto, "A hybrid MAC with dynamic sleep scheduling for wireless sensor networks," 画像電子学会誌, vol. 42, no. 2, pp. 197-205, Feb. 2013.

7. H. Byun and J. Yu, "Adaptive duty cycle control with queue management in wireless sensor networks," IEEE Trans. Mobile Comput. vol. 12, no. 6, pp. 1214-1224, Jun. 2013.

8. W. R. Heinzelman, A. Chandrakasan, and H. Balakrishnan, "Energy-efficient communication protocol for wireless microsensor networks," in Proc. 33rd annual Hawaii International Conference on, System Sciences 2000, pp. 1-10.

9. X. Liu, "An optimal-distance based transmission strategy for lifetime maximization of wireless sensor networks," IEEE Sensors J., vol. 15, no. 6, pp.3484-3491, Jun. 2015.

10. L. Cheng, J. Niu, J. Cao, S. K. Das, and Y. Gu, "Qos aware geographic opportunistic routing in wireless sensor networks," IEEE Trans.Parallel Distrib. Syst., vol. 25, no. 7, pp. 1864-1875, Jul. 2014.

11. W. Su and T. L. Lim, "Cross-layer design and optimization for wireless sensor networks," Int. J. Sens. Networks, vol. 6, no. 1, pp. 3-12, Jan. 2009.

12. T. Kim, D. J. Love, M. Skoglund, and Z.-Y. Jin, "An Approach to Sensor Network Throughput Enhancement by PHY-Aided MAC," IEEE Trans Wirel. Commun., vol. 14, no. 2, pp. 670-684, Feb. 2015.

13. P. Pinto, A. Pinto, and M. Ricardo, "Cross-layer 
admission control to enhance the support of real-time applications in WSN," IEEE Sensors J.,vol. 15, no. 12, pp. 6945-6953, Dec. 2015.

14. P. Park, C. Fischione, A. Bonivento, K. H. Johansson, and A. Sangiovanni-Vincent, "Breath: an adaptive protocol for industrial control applications using wireless sensor networks," IEEE Trans. Mobile Comput., vol. 10, no. 6 , pp. 821-838, Jun. 2011.

15. Z. Lin and M. der Schaar, "Autonomic and distributed joint routing and power control for delay-sensitive applications in multi-hop wireless networks," IEEE Trans. Wireless Commun., vol. 10, no. 1, pp. 102-113, Jan. 2011.

16. W. Ye, J. Heidemann, and D. Estrin, "Medium access control with coordinated adaptive sleeping for wireless sensor networks," IEEE/ACM Trans. Netw., vol. 12, no. 3, pp. 493-506, Jun. 2004.

17. Yuebin B, Shujuan L, Mo S, Yang L, Cong X. "An energy optimization protocol based on cross-layer for wireless sensor networks," Journal of Communications, vol.3, no.8, pp. 27-34, November 2008.

18. T. Winter, "RPL: IPv6 routing protocol for low-power and lossy networks," Internet https://tools.ietf.org/html/rfc6550, March 2012.

19. Munish Gupta, Paramjeet Singh, and Sheveta Rani, "Optimizing physical layer energy consumption for reliable communication in multi-hop wireless sensor networks,", Indian Journal of Science and Technology, vol. 8(13), July 2015.

20. J. A. Haqbeen, T. Ito, M. Arifuzzaman and T. Otsuka, "Intelligent cross layer protocolwith traffic differentiation based QoS for wireless sensor networks," in Proc. 2016 IEEE Region 10 Conference (TENCON), Singapore, 2016, pp. 1088-1092.

21. Y. Liu, I. Elhanany and H. Qi, “An energy-effiecient QoS-aware media access control for wireless sensor networks," in Proc. IEEE Int. Conf. Mobile adhoc sensor System, (TENCON), 2005, pp. 191-193. 